Military Technical College

Kobry Elkobbah,

Cairo, Egypt

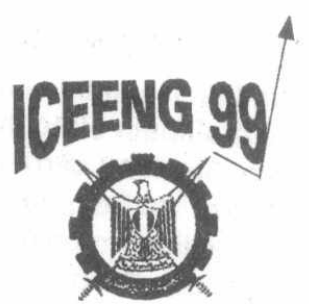

$2^{\text {nd }}$ International Conference

on Electrical Engineering

ICEENG 99

\title{
PHASE-LOCKED LOOP DESIGN FOR MULTIPATH MITIGATION
}

\author{
El-Sayed A. GADALLAH*
}

\begin{abstract}
This paper introduces a new design of Phase-Locked Loop (PLL) that makes receivers with spread spectrum techniques able to work properly in an environment of multipath effect while the receivers with standard PLL is disabled. Basically multipath effect is an inevitable error that can not be removed from the received signal even with the use of a large process gain. The multipath has a dominant effect because the statistical model differs for each possible receiver location.

An investigation of both the standard PLL and the new PLL is introduced. A mathematical approach of the new PLL is presented. Simulation results for both the standard and the new PLL are given. The paper is terminated with conclusions and future work.
\end{abstract}

\section{INTRODUCTION}

PLL plays an important role in establishing coherent references in the receiver carrier-tracking loop. In the presence of multipath, carrier tracking cannot distinguish between the direct and reflected signals and continue to employ null tracking; thus the PLL erroneously estimates the parameters of the direct signal from the transmitter. The PLL aligns a local signal from Voltage Controlled Oscillator (VCO) with the received carrier signal regardless of the shift in its original phase from multipath, Doppler, thermal noise, or other effect. Our approach to multipath mitigation includes three necessary correlated parts: multiple observers of the received signal, an estimator unit, and a modified carrier tracking loop which is the new design of a PLL or what so called the Multiple Reflections PLL (MRPLL) that is the theme of this paper. The estimator unit is accomplished by what so called $\alpha$ deploying estimator [3]. The dynamic performance of the standard PLL to track the received carrier is dependent on the basic characteristic of the phase detector. The slope of such characteristic should be positive when crossing the zero point, namely the equilibrium point. Multipath forces the equilibrium point to move such that the local carrier signal is aligned with the received multipath signal. The received multipath signal is a superposition of the direct signal and other unwanted reflected

* GADALLAH, El-Sayed A. Ph.D., Radar and Guidance Department, Military Technical College 
frequency of the VCO output, $\sin (\varepsilon)$ is often called S-curve. The output of the detector is low pass filtered and provides the control signal that is used to change the frequency of the VCO, for more details about PLL see e.g., $[1,6]$. The multipath effect has no clue to prove its existence on the incoming signal. Thus, the PLL tracks any incoming carrier as a direct she detector S-curve is additional phase added to the intended phase to be measu however there exists a suppressed established crossing the zero phase point (equilibrium notation ' $O$ ' for the direct signal, ' $\wedge$ ' for the bias in the phase due to the multipath. So we use the notation a' at the PLL input and ' $m$ ' for the estimated phase by the PLL, " $p / l$ " for the total infrequency, $R_{\mathrm{c}}$ as the autocorrelation function of multipath, then define: $\omega_{t}$ as the carrer and a ditude, phase, time delay of the Pseudo Random Noise PRN code, and $A, \varphi$, anight summary in Table 1, which shows the PL.L signal, respectively. Thus, we can emplath and 1 illustrates the tracking performance in the cases of absence ans

Table 1: Carrier tracking error in the absence and the presence of the multipath

Table 1: Carrier tracking error in the absence and the presence of the multipath
\begin{tabular}{|l|c|c|}
\hline PLL Parts & In the absence of multipath & In the presence of multipath \\
\hline PLL Input & $\begin{array}{c}A_{o} R_{c}(\delta) \cos \left(\omega_{c} t+\phi_{o}\right) \\
\text { where } \delta=\tau_{o}+\hat{\tau}_{o}\end{array}$ & $\begin{array}{r}R^{\prime}(t)=\sum_{i=0}^{n} A_{i} \cos \left(\omega_{c} t-\phi_{i}\right) \\
\text { where } A_{i}=a_{i} A_{o} R_{c}\left(\delta-\alpha_{i} T_{c}\right) \\
\text { or } \\
R^{\prime}(t)=A_{m} \cos \left(\omega_{c} t+\phi_{o}+\phi_{m}\right)\end{array}$ \\
\hline VCO Output & $\sqrt{2} \sin \left(\omega_{c} t+\phi_{o}\right)$ & $\begin{array}{r}\sqrt{2} \sin \left(\omega_{c} t+\phi_{p l l}\right) \\
\phi_{p l l}=\phi_{o}+\phi_{m}\end{array}$ \\
& $\begin{array}{l}A_{o} R_{c}(\delta) \sin \left(\phi_{o}-\hat{\phi}_{o}\right) \\
\sin \left(\phi_{o}-\hat{\phi}_{o}\right)=\sin (\varepsilon)\end{array}$ & $\begin{array}{l}A_{m} \sin \left(\phi_{p l i}-\hat{\phi}_{p l l}\right) \\
\sin \left(\phi_{o}-\hat{\phi}_{o}+\phi_{m}-\hat{\phi}_{m}\right)\end{array}$ \\
\hline $\begin{array}{l}\text { Tracking Error } \\
\text { S-curve }\end{array}$ & & \\
\hline
\end{tabular}

\section{ANALYSIS AND SIMULATION OF BASIC PLLL}

Analysis and Simulation Modeling of PLL including every component in PLL is accomplished to a which are presented in [2]. The construction of each part is achieved by Simulink ${ }^{\circ}$.

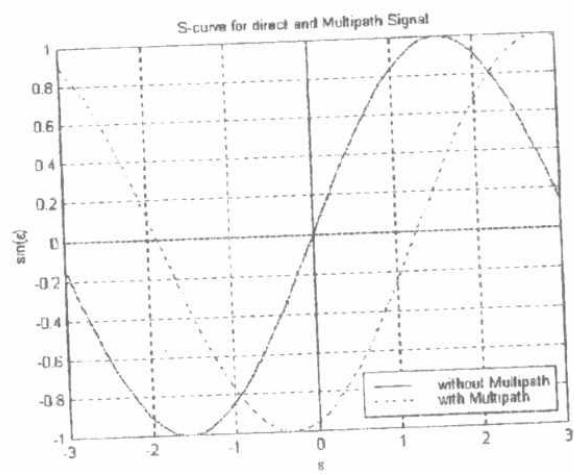




\subsection{VOLTAGE-CONTROLLED OSCILLATOR (VCO)}

It is initially assumed that the loop is operating in the frequency-synchronized mode [7]; that is, only the phase of the VCO must be synchronized with the input signal phase. The input signal is represented as

$$
R_{I P}(t)=A \cos \left(\omega_{c} t+\phi_{o}\right)
$$

And the VCO output is

$$
R_{V C O}(t)=A_{o} \cos \left(\omega_{c} t+\hat{\phi}_{o}\right)
$$

The $\mathrm{VCO}$ is a local signal generator able to change the output phase signal according to the input voltage, $V_{o}$ so as to lock to the reference input phase signal. The oscillator phase change due to this voltage is given by

$$
\frac{d \hat{\phi}_{o}}{d t}=K_{o} V_{o}
$$

where $K_{o}$ is the VCO constant with units rad/sec per volt $[4,8]$. In the notation of the Laplace transformation

$$
\hat{\Phi}_{o}=\frac{K_{o}}{s} V_{o}
$$

In the SIMULINK, Equation 1 and 2 are modeled as elementary math Blocks a sine and a cosine functions, its arguments are the product of ramp function by constant $\left(2 \pi f_{c}\right)$. The phase $\phi_{o}$ can be added to the argument as well. This model is illustrated in Fig. 2 . The input phase to the model can be controlled using slider gain block weighted by constant $(\pi)$. The model shows that the VCO gain $K_{o}$ is equivalent to the unity. The choice of the frequency can be controlled by changing the constant block of $\left(2 \pi f_{c}\right)$. In this simulation we have taken $f_{c}=1 \mathrm{KHz}$.

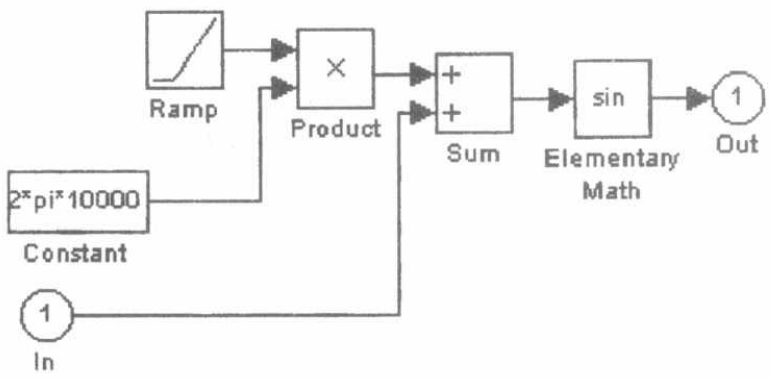

Fig. 2: Voltage-Controlled Oscillator Model

\subsection{PHASE DETECTOR (PD)}

The most important result of this simulation is the modeling of the PD circuits. Actually, the PD circuits determine the types of the PLL, which are analog or digital types. The properties of the PD circuits have a strong influence on the dynamic performance of the PLL system. Four types of PDs are the most frequently used [1], the linear analog computation (four-quadrant multiplier, type1), the digital types (The exclusive-OR gate, type 2, the edge-triggered JK flip flop, type 3, 
and the phase/frequency detector, type (4)). A phase detector is a device whose output is a function of the instantaneous phase difference between two input signals. The defining equation is

$$
V_{o}=K_{d} \sin \left(\phi_{o}-\hat{\phi}_{o}\right)
$$

Where $V_{o}$ is the phase detector output signal, $\phi_{o}$ and $\hat{\phi}_{o}$ are the instantaneous

phase angles of the two inputs, respectively, and $K_{d}$ is the phase detector sensitivity in volts per radian. Phase detectors used in angular feedback loops either of the balanced or the doubly balanced type. The balanced type suppresses one of the input signals, while the doubly balanced type is used when suppression of both input signals is desired. For the mode of operation, with one of two input signals having a much larger amplitude than the other, both the balanced and the doubly-balanced circuits have the same, essentially sinusoidal, input-output relationship [6]. The characteristics of the four PDs types are sinusoidal, triangular, sawtooth and sawtooth with full frequency range, respectively. The type 1 phase detector can be assumed as an ideal multiplier followed by a low-pass filter whose sole effect is to remove the double-frequency component at the multiplier output. The classical digital PLL (DPLL) is not always a pure digital but it is characterized by the appearance of intermediate analog signal. The model we are about to design in this chapter is chosen as the type 1 PLL model. In SIMULINK ${ }^{\theta}$ the multiplier of the phase detector is represented as product block and the low-pass filter is represented as the first order transfer function which is given in Laplace domain as

$$
H_{P D}(s)=\frac{-2 \pi f_{l p f}}{s+2 \pi f_{l p f}}
$$

Where $f_{l p f}$ is the desired cutoff frequency of the LPF of PD. Fig. 3 shows the model of the PD designed in the SIMULINK. The negative polarity is due to the transfer function phase shift.

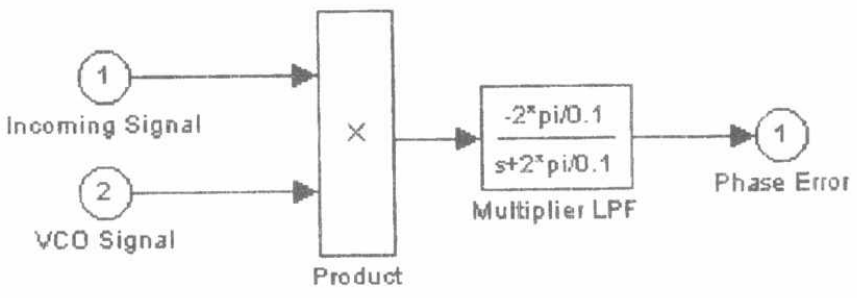

Fig.3: Phase Detector Model

The product of the two PD input signals are the product of the sinusoidal signals defined in Equations, 1 and 2 which is given as

$$
R_{I P} \times R_{V C O}(t)=\frac{1}{2} A A_{o}\left[\sin \left(2 \omega_{c} t+\phi_{o}+\hat{\phi}_{o}\right)-\sin \left(\phi_{o}-\phi_{p l l}\right)\right.
$$

Obviously, the function of the LPF following the multiplier is to remove the first term of second harmonic frequency in Equation 7 and the desired output will be the second term or Equation 5 The speed of the filter and the accepted noise are a trade-off in the PD LPF design. The parameters that influence both the speed and the noise are the filter bandwidth and the magnitude of the filter transfer function at the cut-off frequency of the filter, respectively. The commonly used filters could be a first order integrator, a simple second order system, or a second 
order applying a derivative on the filter input. The corresponding transfer functions for these filters are $\frac{K}{s+a}, \frac{K}{s^{2}+2 \xi \omega_{n} s+\omega_{n}^{2}}$, and $\frac{2 \xi \omega_{n} s+\omega_{n}^{2}}{s^{2}+2 \xi \omega_{n} s+\omega_{n}^{2}}$ respectively.

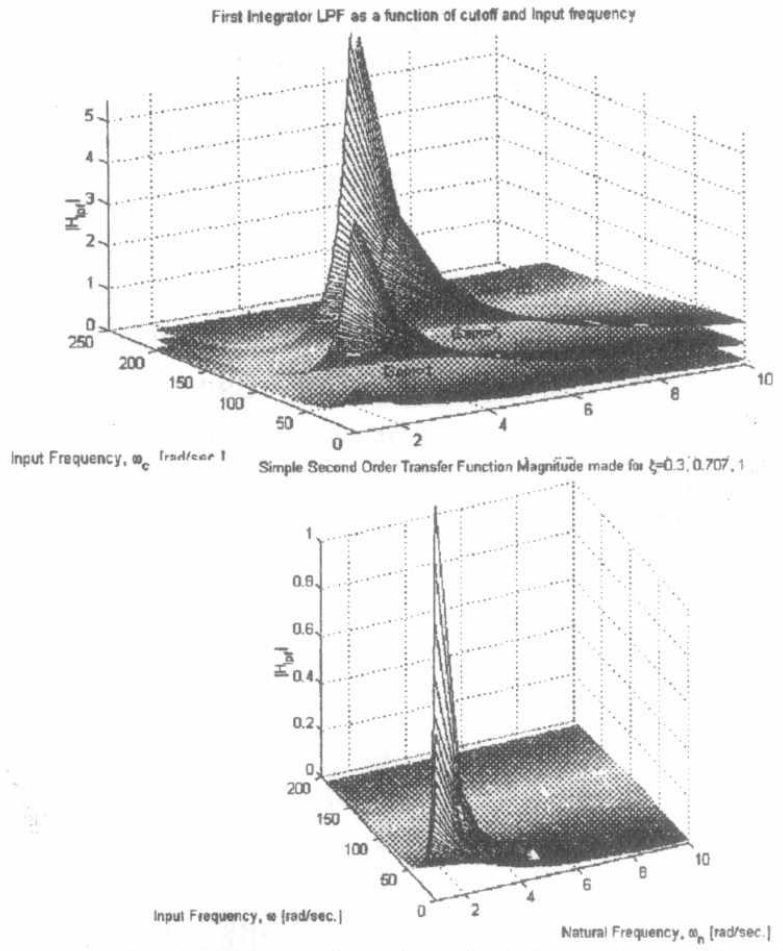

Fig. 4: A typical first order integrator surface LPF gain

Fig. 5: A typical second order LPF surface gain with $\xi=0.3,0.707,1$

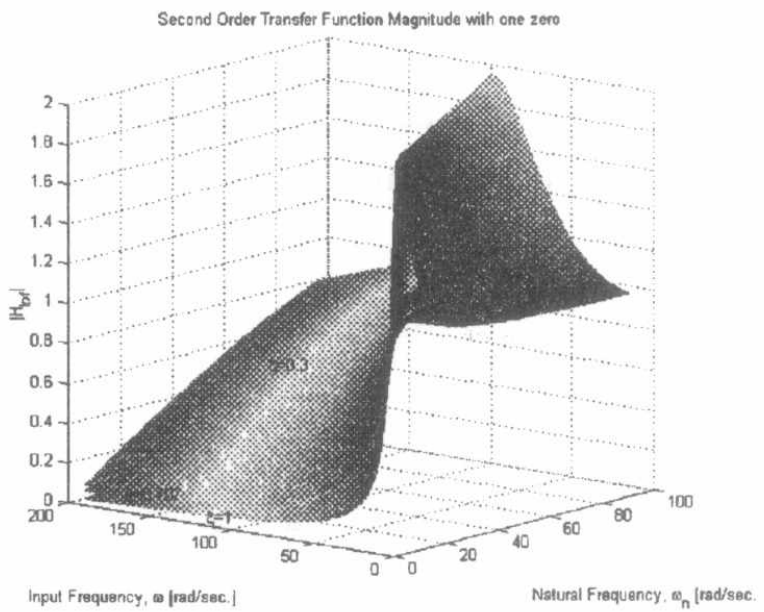

Fig. 6: A typical second order LPF surface gain with one zero with $\xi=0.3,0.707,1$

Fig. 4 shows the magnitude of LPF that has type of first order integrator versus the input angular frequency, $\omega$, and the cutoff frequency, $a$. This figure is symmetric and the filter suppresses high harmonics when the cutoff frequency is getting small, while its magnitude is small which makes its sensitivity small as well. In turn, the filter performance becomes limited. To overcome such disadvantage we increase the gain, $K$, however many harmonics can leak to the output 
filter, which makes its behavior more noisy. Fig. 5 shows the same surfaces plot but for a simple second order. This case is similar to the previous case unless there is a damping factor that influences the filter magnitude instead of increasing the gain, $K$. Fig. 6 shows the filter magnitude to a second order with one zero. The figure shows that as $\xi$ is decreased an increase of gain overshoot is obtained. The main note in this figure is that, the increase of the natural frequency, and in turn the cutoff angular frequency, is followed by the increase of the filter magnitude. Consequently, it means improvement in filter performance. However the tracle-off in the noise harmonics is still there. The numerical values of the LPF bandwidth and the filter magnitude at the cutoff frequency can be adjusted by using the surface curves Fig. 4, 5, and 6 such that the PD output has low noise and the LPF sensitivity is almost unity or greater.

\subsection{PD SIMULATION RESULTS}

The simulation of the PD characteristic can be implemented also by using SIMULINK ${ }^{\circ}$. To show the corruption of the noise into the PD S-curve due to the LPF Bandwidth, $B_{l p f}$, see e.g., Fig. 7 a and $7 \mathrm{~b}$. These figures show that the effect of wider LPF Bandwidth and the increase of L.PF gain cause a leak of some frequency harmonics into the PD output. Thus, the intended constant value of the PD output phase is corrupted with noise. Fig. $8 \mathrm{a}$ and $8 \mathrm{~b}$ show the time simulation of the PD waveforms.

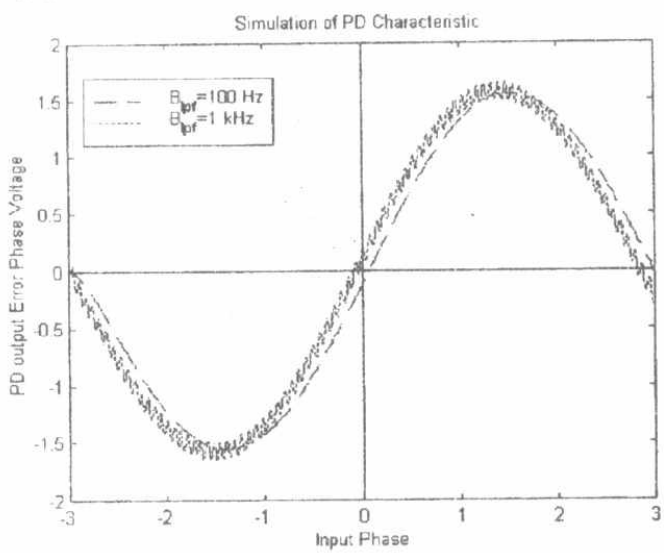

(a)

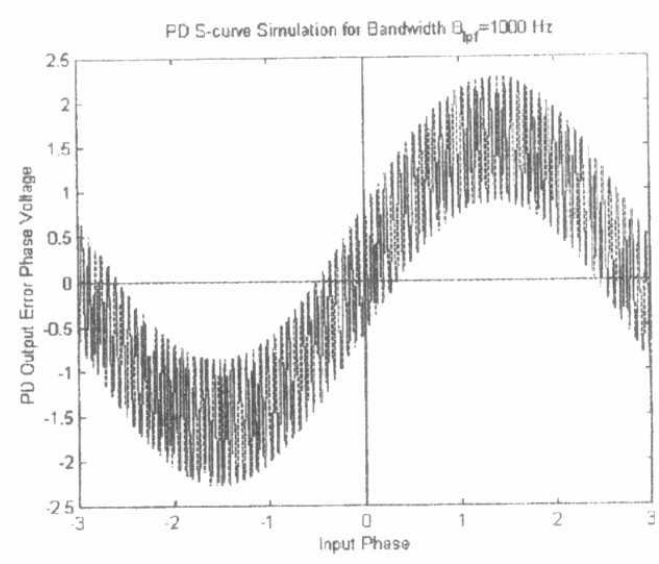

(b)

Fig. 7: On time simulation of the PD S-curve of bandwidth, (a) $B_{l p f}=100 \& 1 \mathrm{kHz}$,

(b) $B_{l p f}=10 \mathrm{kHz}$
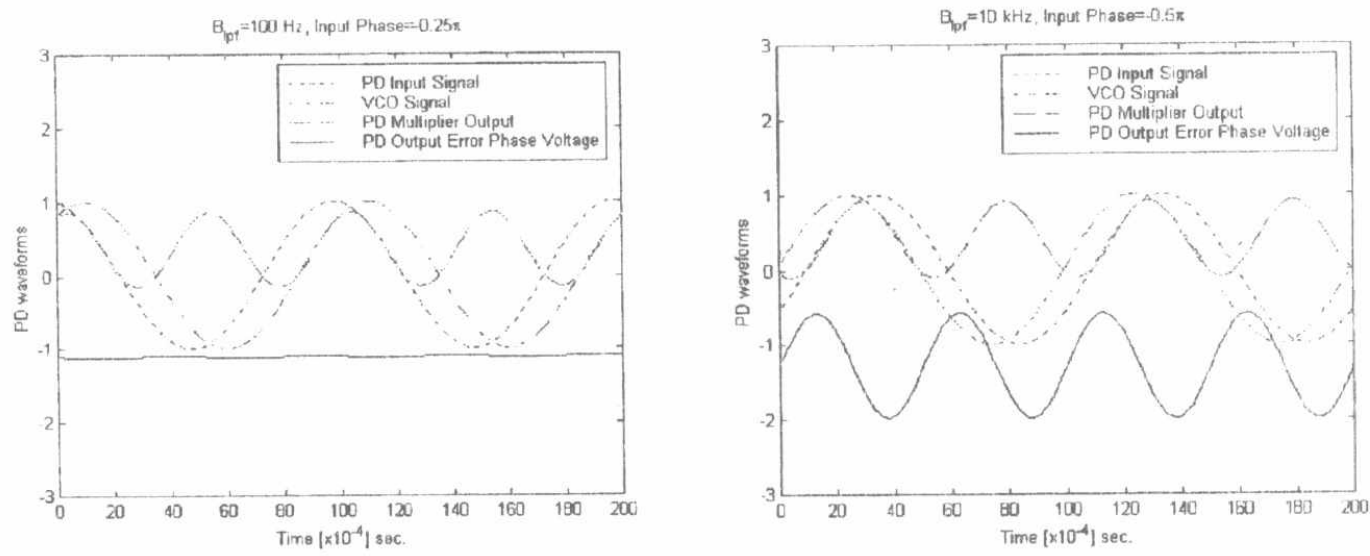

Fig. 8: A simulation scope for PD waveforms of input phase error $=\$-0.25 \pi$ for (a) $B_{l f f}=100$

$\mathrm{Hz} \&$ (b) $B_{l p f}=10 \mathrm{kHz}$ 


\subsection{LINEAR MODEL AND LOOP FILTER}

It is necessary to provide a low pass filter after the PD, to reject carrier frequency components and high frequency noise, which are mentioned in the above section. Thus the dynamic performance of the PLL is influenced not only by the type of PD chosen, but also by the type of loop filter used in particular application. In most cases the loop filter is given by a first and second, order low pass filter. The most general form of transfer function for a first-order filter is realized by lead-lag filter:

$$
F(s)=\frac{a s+b}{c s+d}
$$

In the design of a PLL system we are free to combine any type of PD with any type of realizable loop filter. The possible combination of the first order LPF are taken for, $b=0,(a, b, c, d \neq 0)$, $c=0$, or $b=c=0$. Details of the dynamic response for the PLL system for all of the types of filters and applications are enhancently demonstrated in references $[1,4,8,5$, and 6$]$. The design of the loop filter can be achieved from the dynamic performance analysis of the PLL using the classical control theory. In turn, the PLL transfer function is necessary to be represented in the Laplace domain. The PD characteristic exhibits nonlinearly because the average output signal is a sine function of the phase error, recall Equation 5 . It is approximated that, the most linear part of the $\mathrm{PD}$ characteristic is $\sin (\varepsilon) \approx \varepsilon$, which lets the $\mathrm{PLL}$ to be locked at all times. This approximation part is a quite large region of phase error, which is valid from $-\pi / 3$ to $\pi / 3$ (see [1]). The Phase transfer function is

$$
H(s)=\frac{\hat{\Phi}_{o}(s)}{\Phi_{o}(s)}=\frac{K_{o} K_{d} F(s)}{s+K_{o} K_{d} F(s)}
$$

The order of the PLL system is equal to the order of the loop filter plus 1 [1]. The second order PLLs are the most commonly used, then in order to obtain the phase transfer function of the linear second order PLL, we have to substitute the first order transfer function $F(s)$ of Equation 8 in Equation 9. In this section the loop filter is chosen, $F(s)=\frac{s+a}{s}$. Then we get the transfer function $H(s)$

$$
H(s)=\frac{K_{o} K_{d}(s+a)}{s^{2}+K_{o} K_{d} s+K_{o} K_{d} a}
$$

Equation 10 can be written in the normalized form as

$$
H(s)=\frac{2 \xi \omega_{n} s+\omega_{n}^{2}}{s^{2}+2 \xi \omega_{n} s+\omega_{n}^{2}}
$$

Where $\omega_{n}$ is the natural frequency and $\xi$ is the damping factor. Thus the equivalent substitutions is given by

$$
\begin{aligned}
& \omega_{n}=\sqrt{a K_{o} K_{d}} \\
& \xi=\frac{K_{o} K_{d}}{2 \sqrt{a K_{o} K_{d}}}
\end{aligned}
$$


The term $K_{o} K_{d}$ is called the loop gain and has the dimension $s^{-1}$. If the condition $K_{o} K_{d} \gg \omega_{n}$ is true, this PLL system is said to be a high gain loop [1]. If the reverse is true, the system is called a low gain loop.

Now in the design of the loop filter, we have the constants $K_{o}=1$ and $K_{d}=2 \pi f_{\text {lpf }}$, as seen in the previous section. This implies that $\omega_{n}<<2 \pi f_{l p f}$. Choose $\xi=0.707$, then substitute in Equation 12. Then, the loop filter parameter, $a=\frac{2 \pi f_{l p f}}{4 \xi^{2}}$. So for $f_{l p f}=10 H z F(s)=\frac{s+98.73}{s}$, and, the designed PLL transfer function $H(s)$ is given by

$$
H(s)=\frac{20 \pi(s+98.73)}{s^{2}+20 \pi s+1974.6 \pi}
$$

For more details about the comparison between the analytical and simulation performance of the closed PLL see e.g., [2].

\section{MRPLL, ANALYSIS AND SIMULATION}

The basic idea to mitigate the multipath in this new loop is to force the false equilibrium point of the PD to be in its correct site. Unfortunately the carrier signal is a sinusoidal waveform and the multipath carrier signal is also sinusoidal. So, there is no way to decompose the composite signal of the multipath because the composed components are linearly dependent when they are summed. However, the intelligent idea of the spread spectrum technique aided to solve the problem of the multipath through the coding process. Actually, the decomposition of multipath PRN code is achievable because the summation of the code is linearly independent on each individual multipath component. Nevertheless the front edge of each carrier is related to the front edge of each component of the PRN code. The detail of the multipath parameters is accomplished by using an estimator namely, $\alpha$-deploying estimator [2,3]. The $\alpha$-deploying estimator estimates the number of the reflected signal, $n$, into the direct path signal, the time delay, $\hat{\alpha}_{i}$, for each, $i$, and the amplitude of each component, $\hat{x}_{i}$. So far the multipath phase, $\phi_{m}$, and the multipath amplitude, $A_{m}$ can be calculated in terms of the estimated multipath parameter as:

$$
\begin{aligned}
& \phi_{m}=\arctan \left(\frac{\mathrm{Im}}{\mathrm{Re}}\right) \\
& \hat{A}_{m}=\sqrt{(\mathrm{Re})^{2}+(\mathrm{Im})^{2}}
\end{aligned}
$$

Where

$$
\begin{gathered}
\operatorname{Re}=\sum_{i=0}^{n} \hat{x}_{i} R_{c}\left(\hat{\alpha}_{i} T_{c}\right) \cos \left(2 \pi f_{c} \hat{\alpha}_{i} T_{c}\right) \\
\operatorname{Im}=\sum_{i=0}^{n} \hat{x}_{i} R_{c}\left(\hat{\alpha}_{i} T_{c}\right) \sin \left(2 \pi f_{c} \hat{\alpha}_{i} T_{c}\right)
\end{gathered}
$$

The estimation of multipath parameters are processed and evaluated, then it is

Introduced to MRPLL. MRPLL uses these estimated multipath parameter to cancel the effect of multipath and accomplishes the tracking of the direct signal only. In order to visualize this idea, Fig. 9 shows the MRPLL block diagram proposed to align the estimated carrier phase with the true direct path incoming carrier phase and hence the multipath effect will be canceled. 


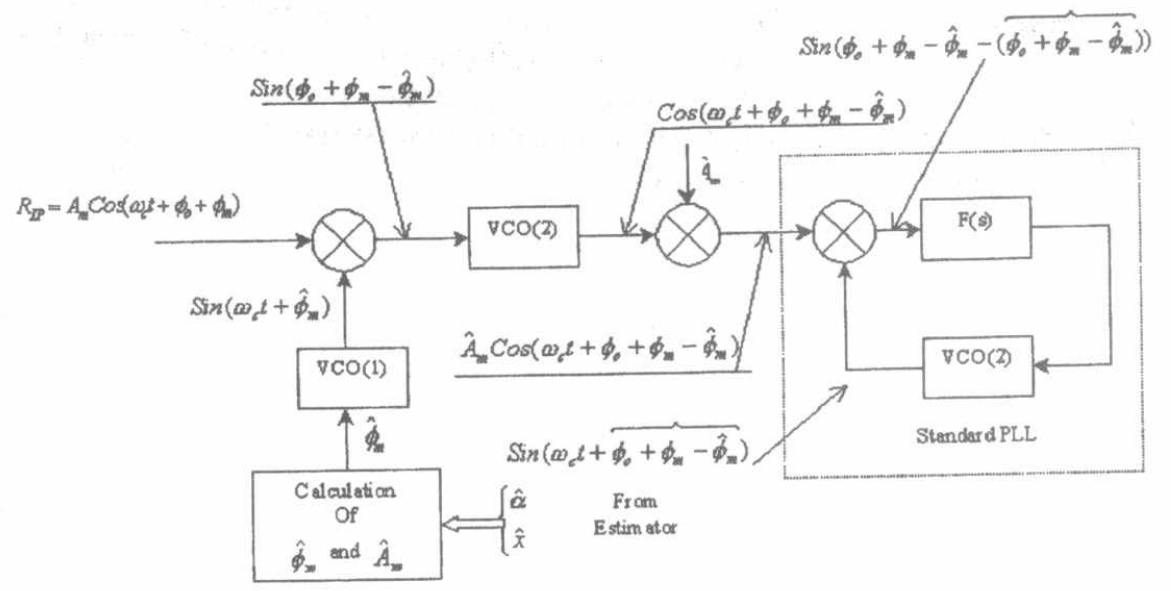

Fig. 9: The MRPLL

The steps for processing the incoming signal in this simulation start with Generating $\operatorname{Sin}\left(\omega_{i} t+\hat{\phi}_{m}\right)$ from VCO (1), whose input is the calculated phase $\hat{\phi}_{m}$ of Equation 14. The first multiplier from the left multiplies the incoming signal with this sine wave, yielding $A_{m} \operatorname{Sin}\left(\phi_{o}+\phi_{m}-\hat{\phi}_{m}\right)$. The VCO (2) generates a cosine signal with phase equivalent to the argument of the sine wave at the input (PD1). The next step is to multiply the cosine signal by the estimated multipath amplitude, $A_{m}$. The actual signal into the PLL becomes

$$
\hat{A}_{m} \operatorname{Cos}\left(\omega_{c} t+\phi_{o}+\phi_{m}-\hat{\phi}_{m}\right)
$$

And the local carrier generated would be

$$
\operatorname{Sin}(\omega_{c} t+\overbrace{\phi_{o}+\phi_{m}-\hat{\phi}_{m}})
$$

Where $\overbrace{\phi_{o}+\phi_{m}-\hat{\phi}_{m}}$ is the phase estimated by the PLL. Therefore, the tracking curve (S-curve) of the PLL can be written from

$$
\operatorname{Sin}(\phi_{o}+\phi_{m}-\hat{\phi}_{m}+\overbrace{\phi_{o}+\phi_{m}-\hat{\phi}_{m}})
$$

The argument of Equation 18 can also be written as

$$
\phi_{o}-\widehat{\phi}_{o}+\phi_{m}-\widehat{\phi}_{m}-\left(\hat{\phi}_{m}-\hat{\hat{\phi}}_{m}\right)
$$

If steady state tracking of the PLL is reached and an exact estimate of $\phi_{m}$ is available, the complete alignment of the direct path signal with the local carrier signal is achievable. A simulation model of the MRPLL is verified versus the standard PLL. The result of the simulation can be summarized in Fig. 10. The incoming composite signal is simulated as a superposition of five sinusoidal components representing the multipath components different in amplitude and phase. These sinusoidal components are added together with the direct signal. The equivalent composite signal, direct signal, and local signal are scoped in Fig. 10 (a) and (b). As soon as a step change is made to the phase of the direct signal, a transition of the tracking signal viz. local one is observed viz. the local signal looses its alignment with the incoming signal. However, it 
goes again to resume the alignment over the incoming signal. Basically the incoming signal in the presence of the multipath is the composite one. Fig. 10 (a) illustrates the time transition of the standard PLL performance where the local signal always goes to align the composite signal whatever the direct path signal phase. While Fig. 11 (b) shows performance of the MF:PLL where it shows the time transition of the local signal until it is aligned with direct signal whatever the phase and the amplitude of the composite signal.

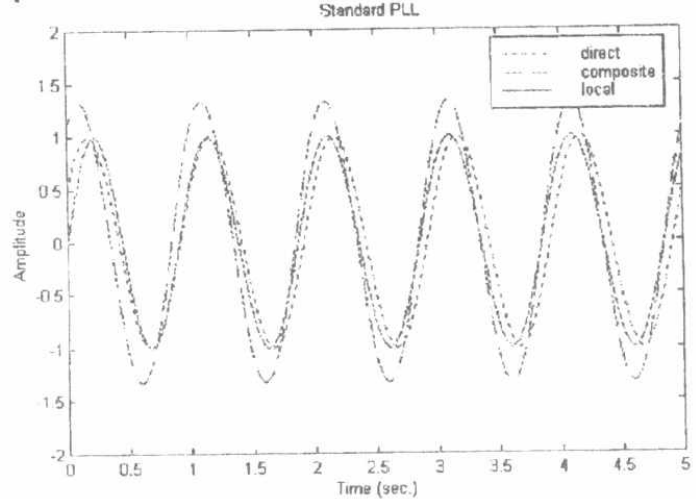

(a)

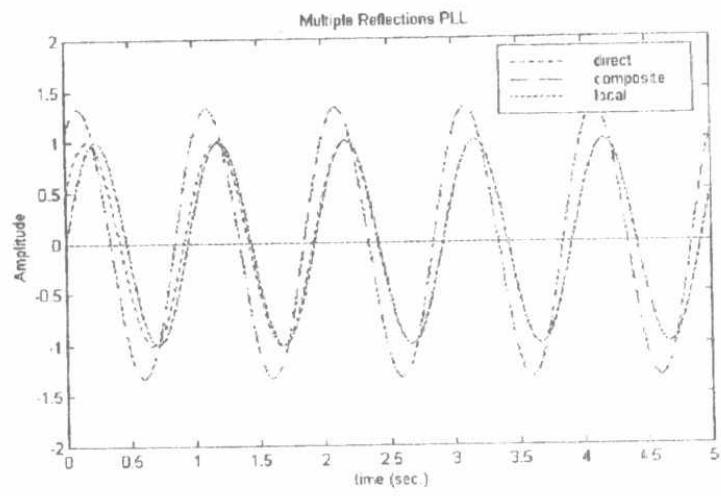

(b)

Fig. 10: on time Simulation of the waveforms of standard PLL and MRPLL

\section{CONCLUSIONS}

In the PD design, it is noticed that the trade-off parameters affecting the PD characteristic versus the PD performance, are the LPF gain and bandwidth. As long as the LPF bandwidth is narrower the accuracy of PD characteristic (S-curve) is improved, however the PD speed performance becomes slower. The MRPLL is necessary to obtain an accurate coherent reference, which is important for the accurate operation of the coherent delay lock loop which is the main active part in the code tracking. The accuracy of the MRPLL depends on the perfect estimation of the multipath parameter. The future work expected includes the noise performance of both the standard PLL and MRPLL and the realization of the simulation model.

\section{REFERENCES}

[1] Roland E. Best. Phase-Locked Loops Theory, Design, and Applications. McGraw-Hill, Inc., NewYork. 1984.

[2] El-Sayed A. Gadallah. Global Positioning System (GPS) Receiver For Multipath Mitigation. Ph.D. Dissertation, Air Force Institute of Technology, WPAFB, OH, 1998.

[3] El-Sayed A. Gadallah. Signal Processing For Multipath Mitigation in GPS. ION Conference, Denver, May 1998.

[4] Floyd M. Garnder. Phaselock Techniques. John Wiley \& Sons, Inc, New York, second edition, 1979.

[5] Leon W. Couch II. Digital and Analog Communication Systems. Macmillan Publishing Co., Inc., New York, 1983.

[6] Jacob Klapper and John T. Frankle. Phase-Locked and Frequency-Feedback Systems. Acadmic Press, New York and London, 1972.

[7] Roger L. Peterson et al. Introduction to Spread Spectrum Communications. Prentice-Hall, Englewood Cliffs, New Jersey, 1995.

[8] W. P. Robins. Phase Noise in Signal Sources. Peter Peregrinus Ltd, London, 1982. 